\title{
Goal Annotation of Process Models for Semantic Enrichment of Process Knowledge
}

\author{
Yun Lin and Arne Sølvberg \\ Dept. of Computer and Information System, Norwegian Univ. of Sci. \&Tech. \\ Sem Sælands vei 7-9, NO-7491, Trondheim, Norway \\ \{yunl, asolvber\}aidi.ntnu.no
}

\begin{abstract}
A semantic annotation framework has been proposed to tackle the semantic heterogeneity problem of distributed process models in our earlier work. The goal annotation as part of the framework is further developed, in which goal ontology is annotated to process models to indicate the objectives or the capability of models. In the paper, we introduce a way to represent goal ontology, build relationships between goals and process models, and develop a goal annotation approach to process models. As an illustration, a case study is deployed with the proposed annotation approach. The results of the goal annotation enrich the semantics of process knowledge from stakeholders perspective in a cooperative goal-oriented manner. The ontology and the annotation results also facilitate the ontology-based queries for the semantic discovery and the reuse of heterogenous process models.
\end{abstract}

\section{Introduction}

As process knowledge, the distributed process models should be accessible and reusable when requesting them for achieving the cooperative business goals. However, those process models were originally created for achieving enterprises local goals. The local goals might be variously presented or even not presented explicitly which causes semantic heterogeneity problem in the goal representation. We need the consensual representations to specify the semantics of goals for the distributed process models, and to enable the machine to interpret and match them to the goal-oriented queries.

The process models involved in this research are the distributed enterprise models on the conceptual level, in which the semantic heterogeneity problem usually occurs. An ontology-based semantic annotation framework is developed to manage the semantic heterogeneity of process models in our previous work [1]. We have provided the approaches and strategies to deal with the semantic heterogeneity of meta-models and model contents. In this paper, the goal annotation based on goal ontology will be refined to complement the semantic annotation framework for facilitating the semantic management of process knowledge.

Goal annotation of process models is annotating process models and model fragments with goal ontology to specify the objectives of processes. Goal ontology is a set of concepts and relationships of semantic definitions about goals. Since the purpose of using goal ontology is to align the semantic representation of goals in a machineunderstandable way, the goals should be represented formally in the goal ontology. 
Based on our objectives and the investigation of several goal modeling methods applied in requirements engineering and process modeling, we propose a representation of goal ontology serving goal-oriented semantic management of process knowledge. Therefore the principles of designing our goal ontology are i) the goal ontology should be process-achievable on the conceptual level, i.e. the goal can be targeted through the process modeling; ii) the corresponding relationships between goal ontology and process model should be easily built to facilitate the goal annotation; iii) the semantics of the goal ontology should be understandable and manipulable for both human and machine.

Goals have been used as an important mechanism for connecting requirements to design and supporting reuse [2]. Goal-driven search of design components [3] and discovery of services [4] uses such kind of mechanism, in which selecting the components or services capable of fulfilling the desired goals (requirements). The goal annotation is a pre-procedure to organize and define the process knowledge with goal ontology, i.e. building relationships between process models and pre-defined goal concepts. The definitions of those relationships are major tasks of the goal annotation, which indicate what relationships are supported in the annotation and how the annotation can be implemented. Consequently, the goal annotated models can be queried for the reuse in a goal-driven method with the goal ontology. The objective of the semantic annotation is to facilitate the knowledge management of heterogenous models via the semantic interoperability.

The goal annotation is accomplished together with other semantic annotations (profile annotation, meta-model annotation and model annotation). Thus, this research work on goal annotation of process models is presented as follows in this paper: we first present our semantic annotation framework to provide the research background of this work; our representation of goal ontology is introduced in section 3; then the corresponding relationships between process models and goal ontology are defined in section 4 ; the procedure of goal annotation accompanied with meta-model and model annotation is described in section 5; and a case study is deployed to illustrate the procedures of goal ontology building and the goal annotation; finally, we conclude the paper and outline the future work.

\section{The Semantic Annotation Framework}

Four main annotation sets constitute the framework: namely, profile annotation, metamodel annotation, model annotation and goal annotation. In the profile annotation, a set of metadata specify the significant characteristics of process models. In the meta-model annotation and the model annotation, we use ontologies to relate constructs across different modeling languages, as well as to align domain specific terminology used in models. Furthermore, the goal annotation is to specify the capacities of process models using a goal ontology. In this way we are able to solve semantic heterogeneity in model management.

A GPO (General Process Ontology) (see Figure 1) is proposed for annotating the process modeling languages in the meta model annotation. Applying the GPO in the meta model annotation, a process model is then described in a PSAM (process semantic annotation model). 


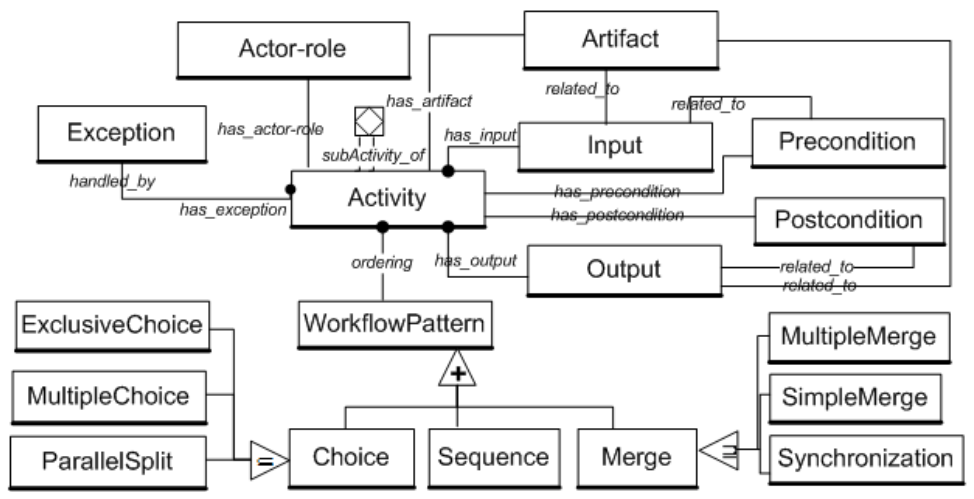

Fig. 1. The GPO

The model annotation and the goal annotation will be employed on the PSAM. The domain ontology and the goal ontology for a certain domain is built or selected by domain experts for the model annotation and the goal annotation respectively. Therefore, a PSAM contains concepts of GPO, domain specific ontology and goal ontology and is defined as follows.

$$
P S A M=\left(A V, A R, A F, W P, I, O, \Theta^{p r e}, \Theta^{p o s} E, P D, P G\right)
$$

Where $A V$ is a set of activities composing a process, $A R$ is a set of actor-roles interacting with a process, $A F$ is a set of artifacts participating in a process, $W P$ is a set of workflow patterns, and each workflow pattern denotes an ordering of activities. $I$ is a set of input parameters, $O$ is a set of output parameters, $\Theta^{p r e}$ is pre-conditions when a process starts, $\Theta^{\text {pos }}$ is post-conditions when a process ends, $E$ is a set of possible exceptions occurring during a process. $P D$ is a subset of domain ontology $(D)$ concepts, i.e. $P D \subseteq D$, including static ontology concepts and task ontology concepts. $P G$ is a subset of goal ontology $(G)$, i.e. $P G \subseteq G$. Since Activity is the main concern in the goal annotation procedure, we provide the annotated activity structure as follows.

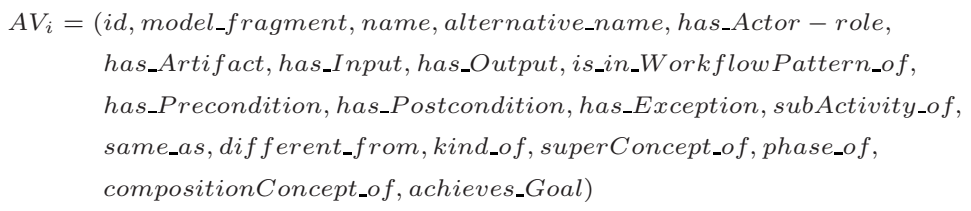

We use $i d$ and model $_{f}$ ragment to locate the annotated model and the original model respectively. The same_as, different_from, kind_of, superConcept_of, phase_of, compositionConcept_of are to annotate the activities with domain ontology, i.e. using semantic relationships mapping an activity with concepts defined in domain ontology. As the goal annotation, the goal ontology is referenced through achieves_Goal. More details refer to [1]. 


\section{Goal Ontology for Semantic Annotation}

A process model represents how to do things not why to do things. Although a process must achieve certain goals, the relationships between goals and processes are not explicitly represented in many process models. With few process modelling language, goals can be modelled and linked to elements of process models, e.g. EEML process and goal models [5]. However, the representations of the goals and the relationships between goals and processes are variously presented in different models. In this work, goal ontology is applied to specify the capability of processes in a consensual way. The focus in this section is modeling goal ontology. We consider three principles to design goal ontology:

- The goal ontology should be process-achievable on the conceptual level, i.e. the goal can be targeted through the process modeling. Thus, the research will not include the goals related to technical factors such as the usage of computing resources or financial aims like reaching a certain amount of gross profit.

- The corresponding relationships between goal ontology and process model should be easily built to facilitate the goal annotation. The process modeling language constructs (e.g. actor, task, resource, ect.) are found overlapped in most existing goal modeling languages such as KAOS [6], i* [7], GBRAM [8] and etc. Accordingly, those goal modeling approaches could be referred to model goal ontology.

- The semantics of the goal ontology should be understandable and manipulable for both human and machine. We use Semantic Web technology — OWL to represent goal ontology. When they are well modeled in OWL Classes and OWL Properties, the model can be interpreted through OWL interpreters.

\subsection{Semantic Representations of the Goal Ontology}

Following the principles, we make a meta-model of the goal ontology considering the semantic expressivity of OWL as Figure 2 In this meta-model, goal ontology are defined based on the goal category and the goal target.

In general, goals can be classified into hard goals and soft goals [9]. Hard goals relate to functional requirements and they are obviously supported by process. Soft goals relate to non-functional requirements (colloquially "-ilities") which are about global qualities of a system. Soft goals are satisfied when there is sufficient positive and little negative evidence for this claim [9]. As the goal category, Hard Goal and Soft Goal are two upper level classes for the goals in all domains. Since hard goals are functional, for different domains they are described domain-specifically. Soft goals can be described generally in a set of "-ilities" (which are regarded as soft goal category), and then specified according to domains.

Checking the literatures of goal definitions, a goal is a condition or state of affairs in the world that stakeholders would like to achieve [10]. Pnina Soffer et al. defines a goal is a set of stable states in [11]. In [12], state of a thing is described as the vector of values for all property functions of a thing. In the context of process modeling, states must be represented as values for the properties of the process and the properties of the objects involved in the process. That is to say, a goal can be expressed as states of 


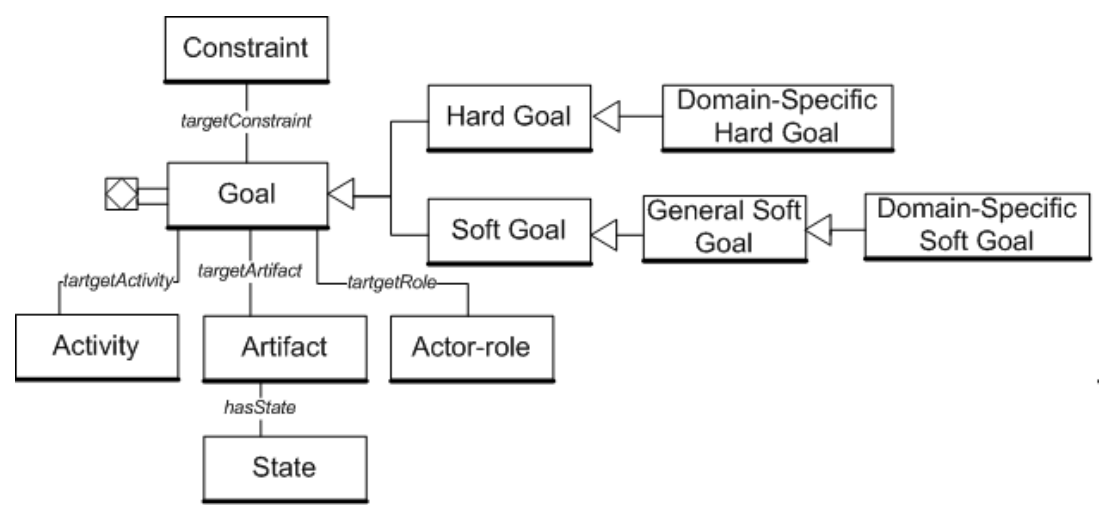

Fig. 2. The meta-model of the goal ontology

activities or states of artifacts. The goal target could be Activity or Artifact. Usually the 'accomplished' is regarded as the goal state of an activity, whilst the state of an artifact has to be specified for different goals. Goal is an organizational concept and goals are held by stakeholders. 'Actor' is defined to represent the goal owner in GRL and 'Agent' is applied in KAOS when analyzing the potential goal realizer. Actor-role is therefore the goal target in the goal ontology as well. In KAOS, goals are non-operational objectives and constraints are operational objectives. Although constraints are not goals, goals can be operated by constraints [6]. In this sense, Constraint is also the goal target. The relationships between Goal and those targets are simply defined because the purpose of the goal ontology is not to analyze the goal like those existing goal modeling methods. The targets show the different perspectives of viewing a goal. These targets are represented same as the concepts in the PSAM, which disclose the potential links between goal ontology and process models.

Decomposition relation - an important characteristic found in most goal analysis should be specified in the goal ontology. However, OWL does not provide any builtin primitives for part-whole relations (as it does for the subclass relation), but contains sufficient expressive power to capture most, but not all, of the common cases [13]. We therefore apply the simple part-whole relationship to represent the decomposition of goal concepts. The 'part' goals contribute the impacts to the 'whole' goal. The logic connections (OR, AND, XOR) between the parts are not considered in the goal ontology due to two reasons. One is the representation limits of OWL. The other one is that the concrete goal analysis mechanism is not necessary for a goal ontology. The goal ontology should be general to applications and how to decompose a goal depends on the specific applications. The goal ontology is more like a taxonomy of goal concepts serving for the semantic-aligned goal representation. The subsumption relationship (owl : subClass) represents the goal category hierarchy. The simple part-whole relationship provides the goal components hierarchy. The terminology presenting goal concepts in the goal ontology should be normalized. Further semantics of a goal are specified through the relationships (owl : objectProperty and owl : dataProperty) with the goal perspectives. 


\section{Relations Between Process Models and Goal Ontology}

We study the relations between process models and goal ontology based on the PSAM model. As we have defined in the GPO in [14], an activity may be an atomic activity or a composite activity which is a synonym of process from this sense. We say that a process model comprises a set of activities $(\boldsymbol{A} \boldsymbol{V})$ and an activity can be decomposed into subactivities. The activities are related to each other through flows according to our GPO definition [1]. If an activity in a process model is not an atomic activity 1 and it is also composed by a set of related activities, it is regarded as a process model fragment in this context. A goal can be linked to a whole process model or to a process model fragment. We assume that the process models are already organized into a decomposable activity hierarchy according to the task ontology in the model annotation phase. Each level activities in a process model can be considered as goal annotation targets.

Definition 1. In the semantic annotation framework, a process model $(P M)$ can be partitioned into several process model fragments (PMF). Each PMF comprises a set of hierachically organized and decomposable $A V$.

Definition 2. Any goal concept $(g)$ in the goal ontology $(G)$ is possibly related to an activity (av) in a PM or PMF:

$\forall(g, a v)$ goalRelated $(g, a v)$

- if the property targetActivity $\left(a v^{\prime}\right)$ of the $g$ is same or synonymic with av:

$\exists\left(a v^{\prime}\right)$ targetActivity $\left(g, a v^{\prime}\right) \bigwedge a v^{\prime}=a v$

- if the property targetArtifact $\left(a f^{\prime}\right)$ of the $g$ is related to the output of av and the State ( $\left.s^{\prime}\right)$ of a $f^{\prime}$ is the value of the Output (o) of the Artifact (af):

$\exists\left(a f, s^{\prime}, o\right)$ targetArtifact $\left(g, a f^{\prime}\right) \wedge$ hasState $\left(a f^{\prime}, s^{\prime}\right) \wedge$

$s^{\prime}=o \supset$ hasOutput $(a v, o) \wedge a f^{\prime}=a f \supset$ relatedTo(o,af $)$

- if the property targetRole ( $\left.a r^{\prime}\right)$ of the $g$ is related to an Actor-role(ar) involved in av:

$\exists\left(a r^{\prime}\right) \operatorname{targeRole}\left(g, a r^{\prime}\right) \bigwedge a r^{\prime}=$ ar $\supset$ hasActor - role $(a v, a r)$

- if the targetConstraint $\left(c^{\prime}\right)$ expressed in the $g$ is involved in (involvedIn) preCondition (pre), postCondition (post) or Exception (e) of av:

$\exists\left(c^{\prime}\right.$, pre, post, e)targetConstraint $\left(g, c^{\prime}\right) \wedge\left(\right.$ involvedIn $\left(c^{\prime}\right.$, pre $) \supset$

hasPrecondition (av, pre) \involvedIn $\left(c^{\prime}\right.$, post $) \supset$

hasPostcondition (av, post) \involvedIn $\left(c^{\prime}, e\right) \supset$

hasException $(a v, e))$

Therefore,

$(a) \equiv(b) \bigvee(c) \bigvee(d) \bigvee(e)$

${ }^{1}$ Note: An atomic activity can not be decomposed, but it is not an event either. 
Checking above cases through matching algorithms can automatically provide a list of possible goal annotations. The decisions of the desired goal annotations are left to annotators.

Definition 3. In the goal ontology $(G)$, the hard goal set is notated as $G^{h}$ and the soft goal set is notated as $G^{s}$.

The relations are further specified by the annotator based on the context of the process models and the goal ontology. We define two relations as follows:

Definition 4. Hard goals can be achieved by an activity or activities. I.e. the relation between the activity (av) and the hard goal $\left(g^{h}\right)$ is achieves $\left(a v, g^{h}\right)$.

Definition 5. Soft goals can be positively or negatively satisfied by an activity or activities. I.e. the relation between the activity (av) and the soft goal $\left(g^{s}\right)$ is positivelySatisfies $\left(a v, g^{s}\right)$ or negativelySatisfies $\left(a v, g^{s}\right)$.

Since the activities in the process models are decomposable, the relation between goals and a composite activity could be inferred based on the relations between goals and component activities.

Definition 6. If an activity (av) is a component of another activity $\left(a v^{\prime}\right)$ in a process model/model fragment, av is the subactivity of $a v^{\prime}$, i.e. subActivityOf $\left(a v, a v^{\prime}\right) . a v^{\prime}$ is a Composite Activity in that model.

Usually the effects of hard goals achieved by a subActivity can contribute to its composite activity. That is,

Definition 7. If av is the subactivity of $a v^{\prime}$ and av achieves $g^{h}$, av achieves $g^{h}$ :

$$
\begin{aligned}
& \left(\forall\left(a v, g^{h}\right) \exists a v^{\prime}\right) \text { subActivityOf }\left(a v, a v^{\prime}\right) \wedge \operatorname{achieves}\left(a v, g^{h}\right) \\
& \longrightarrow \text { achieves }\left(a v^{\prime}, g^{h}\right)
\end{aligned}
$$

However, the effects of soft goals can not be simply passed in the same way as hard goals. To a composite activity, the contribution of a soft goal from a subactivity might be enhanced or reduced by other subactivities which positively or negatively satisfy the same soft goal. The contribution could be calculated if the effects of soft goals are quantified. This issue is only simply considered in our current work by simple contribution calculation rules. All effects of soft goals are regarded same. The contribution of soft goals to a composite activity is determined by comparing the numbers of subactivities which positively satisfy and negatively satisfy the same soft goals. That is,

Definition 8. Let a $g^{s}$ is positively satisfied by $N$ subactivities, and is negatively satisfied by $M$ subactivities in a composite activity av',

- if $N>M$, positivelySatisfies $\left(a v^{\prime}, g^{s}\right)$

- if $N=M$, then the effect of $g^{s}$ is counteracted for $a v^{\prime}$.

- if $N<M$, negativelySatisfies $\left(a v^{\prime}, g^{s}\right)$

The relations between goals and activities defined in this section will be applied to build annotation links between goal ontology and process models in the goal annotation. That is to say, the meta data schema of the goal annotation for process models is: 


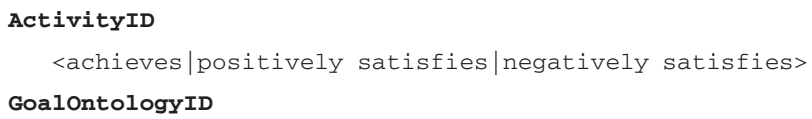

\section{Goal Annotation Procedure}

In the goal oriented requirements engineering, the goal analysis and modeling is a top to bottom procedure - decomposing high level goals down to lower level goals and operational activities. The goal annotation is a bottom to top procedure - first annotating low level subactivities and then annotating higher level activities and the whole process model with goal ontology. The goal annotation is employed based on the domain annotated PSAM models.

After the profile annotation, the PSAM is initially structured after the meta-model annotation, and then it is gradually filled with domain ontology in the model annotation. The goal annotation is employed as the final step of the whole annotation procedure, i.e. filling the PSAM with goal ontology. The semantic annotation procedure is illustrated in Figure 3 .

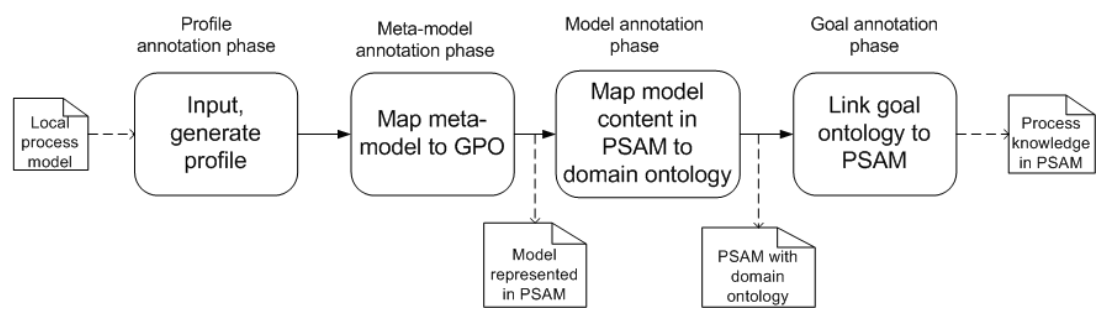

Fig. 3. The semantic annotation process based on PSAM

As the goal annotation, we update the PSAM with the goal annotation relations achieves_Goal/positively_satisfies_Goal/negatively_satisfies_Goal . Applying the psam as markup annotation language, an example of annotating a process model fragment with the goal ontology by representing an activity achieves a hard goal as follows:

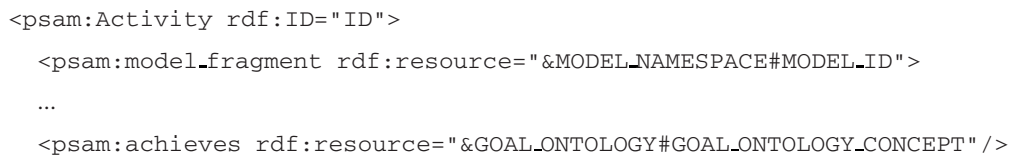

We have discussed that the goal annotation of the process models and the model fragments is to link goal ontology to the activities identified in the models. Focusing on the activity, we describe the goal annotation procedure accompanied with the meta model annotation and the model annotation in a UML activity diagram in Figure 4.

Through the meta model annotation, activities are identified by the markup Activity in a PSAM. In the model annotation phase, if the domain task ontology is available 


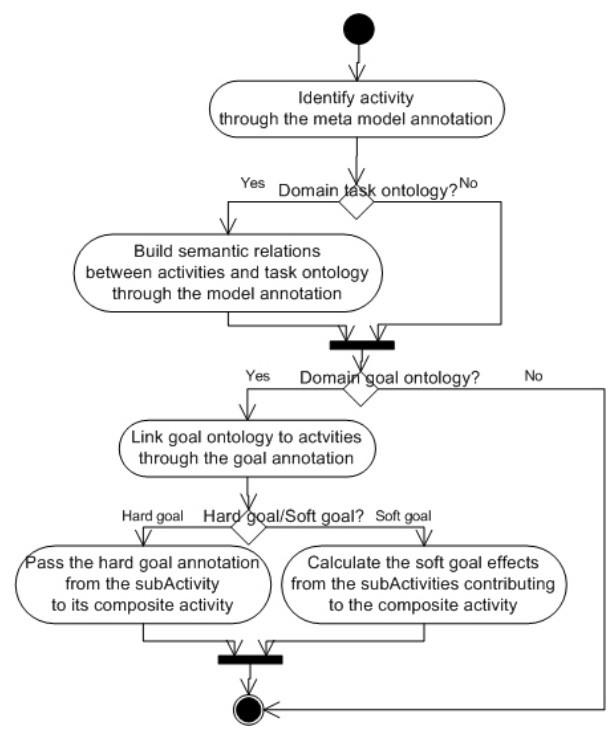

Fig. 4. The goal annotation procedure

as the activity references, the identified activities is annotated with the task ontology references via the semantic relations such as same_as, different_from, kind_of, superConcept_of, phase_of, and compositionConcept_of. If the domain goal ontology is available, the possible links between the activities and the goal ontology can be checked based on the relations described in section 4 . We employ the annotation from the component activities to the composite activities. The contributions of the goals annotated to the low level component activities can be passed to or calculated for their upper level composite activities.

\section{Case Study}

In this section, we provide a case study to illustrate the goal ontology representation and the goal annotation procedure. For the sake of the brevity, we assume the models have already processed by the meta model annotation and the model annotation. Consequently, the goal annotation is employed on the PSAM model. We set our example in an industrial enterprise with supply-chain management systems. The SCOR (Supply Chain Operations Reference-model) [15] is applied as the domain ontology in this example. The goal ontology is also derived from the SCOR.

\subsection{The TelCo Case Study}

TelCo company is specialized in telecommunications, in the production and distribution of batteries, as well as in retail sales of everyday technology products. TelCo does not have its own warehouse but uses the services of logistics company Orbit Ltd. But 


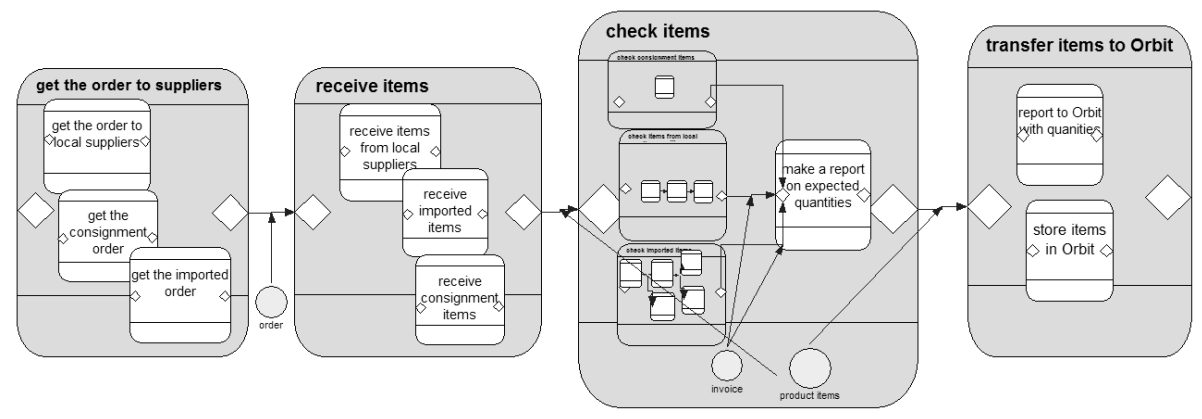

Fig. 5. TelCo item receiving process

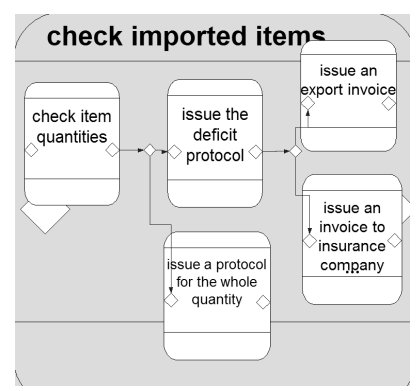

Fig. 6. Decomposition of the check items

TelCo has its logistics department who is responsible for items receiving and delivering. Thus the functions of logistics department are to order and control Orbit. The following main business processes of the logistics are items receiving, returns, orders from shops and franchisees, orders from dealers and inventories. In this case, we take the items receiving process as the annotation example. The process model of the items receiving is initially made in EEML. A simplified model mainly from the task viewpoint is displayed in Figure 5. The logistics department receives a report for expected quantities of items according to the order to the supplier, and prepares to receive the items. After receiving the items, the logistics department checks items. Sub-tasks are included in some tasks because there are three types of items receiving - regular orders to local suppliers, consignment and import. The process details are described in the decomposition of the task check import items in Figure 6, After checking the items, the received items will be transferred to Orbit. Meanwhile the department sends a report to Orbit in order to inform them what to expect.

The meta model annotation is to map the EEML meta model and the model structures to the GPO. Hereby, all the tasks in the EEML model are annotated as Activity in the PSAM. For the model annotation and the goal annotation, the SCOR is referenced as the domain ontology including the domain tasks and the domain goals. 


\subsection{The SCOR as Reference Ontology}

The SCOR is a process reference model that has been developed and endorsed by the Supply-Chain Council as the cross-industry standard diagnostic tool for supply-chain management. The SCOR-model describes the business activities associated with all phases of satisfying a customer's demand.

For this case, we apply "S1 Source Stocked Product" model as task ontology for the item receiving process. The SCOR-model is depicted in Figure 7 Each process element is a task ontology concept which is referenced by the Activity in the model annotation (e.g. the Activity check items is annotated with the task ontology Verify Product). The inputs and outputs are the domain object concepts as ontology to annotate the Artifacts in the PSAM (e.g. the Artifact product item is annotated with the domain object ontology Sourced Product On Order). The details of the model annotation are not specified in this paper due to the space limitation.

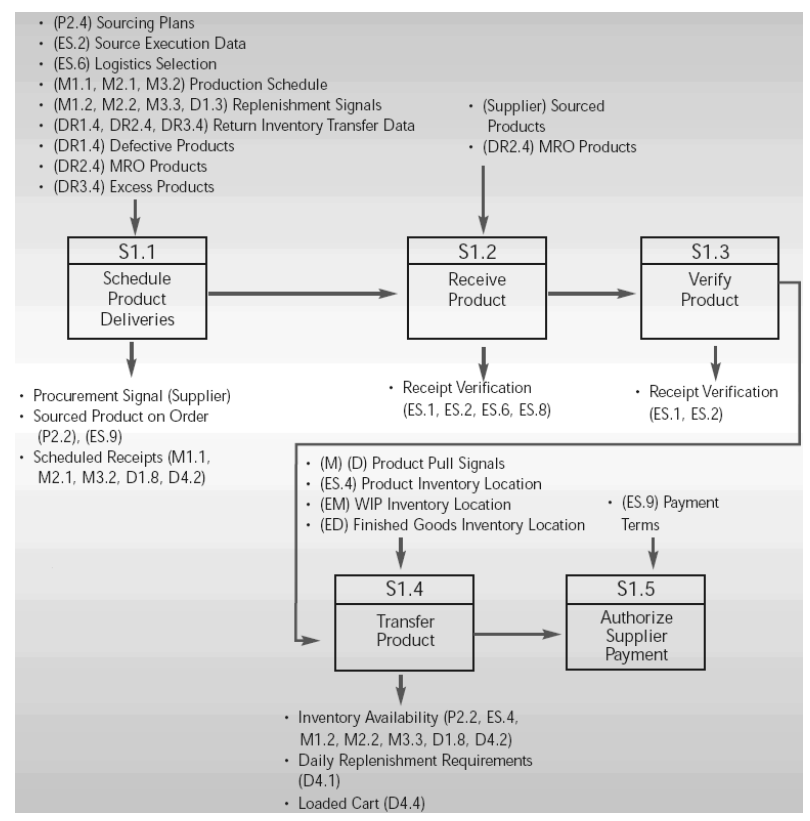

Fig. 7. S1 Source Stocked Product

The goal ontology in this domain is also from the SCOR. Usually the hard goals are derived from the level 3 process elements [15] and their inputs and outputs. The performance attributes defined in SCOR are General Soft Goal Category (generally in a set of "-ilities") such as Reliability,Responsiveness, Flexibility, Cost, and Assets. The domain specific soft goals are derived from the metrics of the performance attributes [11]. By analyzing the goal targets, we can identify the following goals derived from S1 (Table 1). 
Table 1. Goal ontology derived from SCOR

\begin{tabular}{|c|c|c|}
\hline Goal Type & Goal Target & SCOR Goal Ontology \\
\hline \multirow{3}{*}{ Hard Goal } & targetActivity & sourced products are transferred; sourced products are verified. \\
\hline & targetArtifact and state & sourced products on order; scheduled receipt; available inventory. \\
\hline & targetRole & payment is authorized to supplier \\
\hline \multirow[t]{3}{*}{ Soft Goal } & targetConstraint & \begin{tabular}{|lcccr} 
improve supplier & delivery & to & date & performance \\
(Responsiveness); & invoices & processed & without & error \\
(Reliability). & & & & \\
\end{tabular} \\
\hline & targetArtifact & $\begin{array}{l}\text { improve delivery quantity performance (Assets); decrease \% de- } \\
\text { fective supplied (Reliability). }\end{array}$ \\
\hline & targetActivity & $\begin{array}{l}\text { reduce verification costs (Costs); reduce receiving \& storage } \\
\text { costs (Costs). }\end{array}$ \\
\hline
\end{tabular}

\subsection{Goal Annotation for Semantic Enrichment of Process Knowledge}

Having a set of goal ontologies in the Sourced Stocked Product domain, we annotate the TelCo logistics department's item receiving process model. We consequently have the following annotation results listed in Table 2 .

The orginial EEML tasks are listed in the first column. After the meta-model and the model annotation, each task is represented as a PSAM:Activity and is linked to SCOR domain ontology through the semantic relationships (refer to the second column). The goal annotation is to build a link from the goal ontology to the PSAM activities. The annotation relations in third column are defined in section 4 SCOR goal ontology in the fourth column from table 1

Table 2. Goal annotation results

\begin{tabular}{|c|c|c|c|}
\hline EEML Tasks & $\begin{array}{l}\text { PSAM Activities (Model annota- } \\
\text { tion with SCOR domain ontology) }\end{array}$ & $\begin{array}{l}\text { Goal Annotation } \\
\text { Relations }\end{array}$ & SCOR Goal Ontology \\
\hline get the order to supplier & $\begin{array}{l}\text { phase_of Activity:Schedule Product } \\
\text { Deliveries }\end{array}$ & achieves & sourced product on order \\
\hline get the order to supplier & $\begin{array}{l}\text { phase_of Activity:Schedule Product } \\
\text { Deliveries }\end{array}$ & positively_satisfies & \begin{tabular}{|llll}
$\begin{array}{l}\text { reduce } \\
\text { costs }\end{array}$ & receiving $\&$ & storage \\
\end{tabular} \\
\hline \begin{tabular}{|l|} 
check imported items \\
\end{tabular} & kind_of Activity:Verify Product & positively_satisfies & $\begin{array}{l}\text { improve delivery quantity per- } \\
\text { formance; decrease } \% \text { defec- } \\
\text { tive supplied }\end{array}$ \\
\hline \begin{tabular}{|l} 
check imported items \\
\end{tabular} & kind_of Activity:Verify Product & negatively_satisfies & reduce verification costs \\
\hline $\begin{array}{|ll|}\text { check } & \text { consignment } \\
\text { items } & \end{array}$ & kind_of Activity:Verify Product & positively_satisfies & reduce verification costs \\
\hline transfer items to Orbit & kind_of Activity:Transfer Product & achieves & available inventory \\
\hline $\begin{array}{l}\text { issue the invoice; issue } \\
\text { an export invoice }\end{array}$ & $\begin{array}{l}\text { phase_of Activity:Authorize Sup- } \\
\text { plier Payment }\end{array}$ & achieves & $\begin{array}{l}\text { payment is authorized to sup- } \\
\text { plier }\end{array}$ \\
\hline $\begin{array}{l}\text { issue the invoice; issue } \\
\text { an export invoice; issue } \\
\text { an invoice to insurance } \\
\text { company }\end{array}$ & $\begin{array}{l}\text { phase_of Activity:Authorize Sup- } \\
\text { plier Payment }\end{array}$ & negatively_satisfies & $\begin{array}{l}\text { invoices processed without er- } \\
\text { ror }\end{array}$ \\
\hline
\end{tabular}

The EEML task get the order to supplier can be regarded as a phase of the activity ontology Schedule Product Deliveries from SCOR. When annotating goals, we find two goals in the domain goal ontology related to this activity. It can achieve a hard goal sourced product on order and also positively satisfy a soft goal reduce receiving \& storage costs. Three kinds of items receiving are checked through the activity check items. The procedure of check imported items 
is a little more complicated compared with the other two kinds of items receiving becuase it includes issuing deficit protocols and an invoice to insurance company. It therefore negatively satisfies the soft goal reduce verification costs. However, this check procedure can improve the delivery quantity performance and the deficit check can ensure the low \% defective supplied. The consignment can simplify the check and item receiving procedure, so it positively satisfies the soft goal of reduce verification costs. To transfer items to Orbit is a procedure of Transfer Product and the result of the procedure is an available inventory. Issuing invoices are steps of Authorize Supplier Payment, in which issue the invoice to local suppliers and issue an export invoice are to authorize payment to suppliers. The soft goal of invoices processed without error will be risked by different invoice issue procedures.

After annotating the low level activity elements, the goal contributions can be calculated to the upper level activities. Taking the example of the composite activity check items, we have annotated its component activities with hard goals and soft goals. Check imported items negatively satisfies reduce verification costs and check consignment items positively satisfies reduce verification costs, so the effects are counteracted for the composite activity check items if we apply the simple contribution calculation rules. Without negative counteraction, the soft goals improve delivery quantity performance and decrease \% defective supplied contribute themselves to check items. The hard goals annotated to issue the invoice and issue an export invoice are also simply passed to check items. The soft goal invoices processed without error are negatively satisfied by check items and the effects are enhanced due to three component activities negatively satisfy this soft goal.

\subsection{Process Knowledge Discovery and Reuse Based on the Semantic Annotation}

Before the semantic annotation, the local process models are represented in EEML and the model contents are described with the concepts and terms locally defined, such as 'items', 'supplier', 'check items'. The EEML might not be acquainted to other users who want to reuse the model. The users who want to search the model do not know the exact concepts and terms used in the model, if applying the keyword search. Usually, users want to find the model which can realize their goals. However, the goals usually do not be specified in the local process models or specified in the modeling languages strange to users.

The proposed semantic annotation approach makes the process knowledge of TelCo logistics department explicit and open to the third party who is interested in the model exchange, system integration and business cooperation in the SCOR domain. An external party would like to get the knowledge from the existing models, which include this EEML model and other heterogenous models in different modeling languages. She can make search based on the SCOR goal and domain ontology. If she provides a business goal which is represented in the SCOR goal ontology, the query will be matched to the goal annotated process models. For example, the user would like to check the existing models/model fragments which impact the soft goal reliability in the delivery process. In the goal ontology, the soft goal concept 'Reliability' has some 
sub-classes such as 'Improve Delivery Performance', 'Improve Fill Rates' and 'Improve Perfect Order Fulfillment'. The user might decide to only take the goal 'Improve Delivery Performance' as the query. The mechine makes the match and the semantic inference between the query and the goal annotation in the process models. In the query results, we find not only the process model fragments annotated with the goal concept 'Improve Delivery Performance', also those annotated with the goals 'Improve supplier delivery to date performance' and 'Improve delivery quantity performance', because the goals 'Improve supplier delivery to date performance' and 'Improve delivery quantity performance' are part goals of 'Improve Delivery Performance' in the SCOR goal ontology. The models/model fragments annotated with the corresponding goal ontology will be returned to the user as a result.

The returned results include the original models and the annotation information. Suppose the users do not know EEML, but they know the GPO. Compared with a certain modeling language, GPO provides much simpler structures but enough core process semantics. The meta-model annotation provides the mapping relations between the EEML and the GPO. Also from the PSAM of a model, users can read the mapping between the local concepts in the original model and the domain ontology concepts. We assume that users well know the domain ontology as the domain standard. Consequently, the model annotation helps the users to understand the local concepts, which is required in the later model reuse and model transformation.

\section{Conclusions and Future Work}

As part of the semantic annotation framework, a goal annotation approach is elaborated in this paper. The semantic annotation framework is designed for managing the semantic heterogeneity of distributed process models on the conceptual level. The approach is based on the domain ontology, which provides common semantic representations of domain concepts. The semantic annotation framework aligns the semantic heterogeneity of models from different perspectives. The purposes of the goal annotation are two: 1) to enrich the semantics of the objectives of processes; 2) to provide a way to find the process knowledge based on business goals. Goal ontology is necessary for the goal annotation as the common semantic representations of process objectives. Based on the existing goal modeling methods, we proposed a way to describe the semantics of goals for the process model annotation purpose. We also discuss the relations and rules between process models and goal ontologies. The formulization of the relations can be implemented to facilitate the automatic or semi-automatic goal annotation procedure in practice. The proposed approaches are examined through a case study. Main work includes the establishment of a domain goal ontology from SCOR and the execution of the goal annotation based on the relations and rules. The possible applicability of the annotation result is also briefly presented.

The proposed goal ontology semantics and goal annotation relations can be further elaborated. More semantics are not explicitly represented and enriched, for instance, the contribution relationships between goals ('support', 'conflict', etc.), and the impact degree between activity and goal ('partially impact', 'totally impact'). The tools for the 
automatic annotation and the visualization of annotated models are also the challenges in this research.

\section{Acknowledgement}

This work is partially supported by the Norwegian Research Foundation in the framework of Information and Communication Technology (IKT-2010) program. Also thanks the INTEROP project for the case study.

\section{References}

1. Lin, Y., Strasunskas, D., Hakkarainen, S., Krogstie, J., Sølvberg, A.: Semantic annotation framework to manage semantic heterogeneity of process models. In: Dubois, E., Pohl, K. (eds.) CAiSE 2006. LNCS, vol. 4001, pp. 433-446. Springer, Heidelberg (2006)

2. Yu, E., Mylopoulos, J.: Why goal-oriented requirements engineering. In: Proc. of the 4th of International Workshop on Requirements Egnineering: Foundations of Software Quality (1998) http: / /www.cs.toronto .edu/pub/eric/REFSQ98.html

3. Hummel, K.A., Jochum, W., Leitich, S., Schandl, B.: Supporting meetings with a goal-driven service-oriented multimedia environment. In: MSC '05: Proceedings of the first ACM international workshop on Multimedia service composition, pp. 55-65. ACM Press, New York (2005)

4. Lin, M., Guo, H., Yin, J.: Goal description language for semantic web service automatic composition. In: Proc. of IEEE/IPSJ International Symposium on Applications and the Internet (SAINT 2005), 31 January - 4 February 2005, Trento, Italy. pp. 190-196 (2005)

5. Krogstie, J., Jørgensen, D.: Interactive models for supporting networked organisations. In: Persson, A., Stirna, J. (eds.) CAiSE 2004. LNCS, vol. 3084, pp. 550-562. Springer, Heidelberg (2004)

6. Dardenne, A., van Lamsweerde, A., Fickas, S.: Goal-directed requirements acquisition. Science of Computer Programming 20, 3-50 (1993)

7. Yu, E.: $i^{*}$ :an agent-oriented modelling framework (2006) http://www.cs.toronto.edu/km/istar/

8. Anton, A.I.: Goal based requirements analysis. In: Proc. Second Int. Conference on Requirements Engineering, ICRE 96. pp. 136-144 (1996)

9. Mylopoulos, J., Chung, L., Yu, E.: From object-oriented to goal-oriented requirements analysis. Commun. ACM 42, 31-37 (1999)

10. Grl, G.R.L.: ontology ( 2007) http: / / www . cs . toronto.edu/km/GRL/

11. Soffer, P., Wand, Y.: On the notion of soft-goals in business process modeling. Business Process Management Journal 11, 663-679 (2005)

12. Wand, Y., Weber, R.: On the deep structure of information systems. Information System Journal 5, 203-223 (1995)

13. W3C: Simple part-whole relations in owl ontologies (2005) http://www.w3.org/ 2001 /sw/BestPractices/OEP/SimplePartWhole/index.html

14. Lin, Y., Strasunskas, D.: Ontology-based semantic annotation of process models. In: Proc. of 10th CAiSE/IFIP8.1/EUNO International Workshop on Evaluation of Modeling Methods in System Analysis and Design (EMMSAD05) Porto, Portugal (June 2005)

15. SCOR: Scor model (2006) http://www.supply-chain.org/page.ww?section=SCOR+Model\& name $=$ SCOR + Model 